\title{
Quantum permanents and Hafnians via Pfaffians
}

\author{
Naihuan Jing and Jian Zhang
}

\begin{abstract}
Quantum determinants and Pfaffians or permanents and Hafnians are introduced on the two parameter quantum general linear group. Fundamental identities among quantum Pf, Hf, and det are proved in the general setting. We show that there are two special quantum algebras among the quantum groups, where the quantum Pfaffians have integral Laurent polynomials as coefficients. As a consequence, the quantum Hafnian is computed by a closely related quantum permanent and identical to the quantum Pfaffian on this special quantum algebra.
\end{abstract}

\section{Introduction}

In revealing the correspondence between the bosonic and fermionic versions of the Wick formula, Caianiello [C] introduced the Hafnian of a symmetric matrix $C$ of even dimension as

$$
\operatorname{Hf}(C)=\frac{1}{n !} \sum_{\sigma \in \Pi^{\prime}} c_{\sigma(1) \sigma(2)} c_{\sigma(3) \sigma(4)} \cdots c_{\sigma(2 n-1) \sigma(2 n)},
$$

where $\Pi^{\prime}$ consists of all permutations $\sigma \in S_{2 n}$ such that $\sigma(2 i-1)<\sigma(2 i), i=$ $1, \ldots, n$. It is known that the Hafnian has several properties similar to the Pfaffian [LT]. We will add one more identity $\operatorname{Hf}(C)=\operatorname{per}(A)$ for a closely related matrix $A$ (cf. Proposition 3.7), which appears to be fundamental as one notices that $\operatorname{Hf}(C) \neq \sqrt{\operatorname{per}(C)}$ in general. Here the permanent $\operatorname{per}(C)$ C] is defined by changing all the signs to +1 in $\operatorname{det}(C)$, and sometimes also referred as the positive determinant $[\mathbf{M}]$.

In [JZ], we have defined the quantum Pfaffian of an even dimensional matrix $B=\left(b_{i j}\right)$ with noncommutative entries by

$$
\operatorname{Pf}_{q}(B)=\frac{1}{[n]_{q^{4}} !} \sum_{\sigma \in \Pi^{\prime}}(-q)^{l(\sigma)} b_{\sigma(1) \sigma(2)} b_{\sigma(3) \sigma(4)} \cdots b_{\sigma(2 n-1) \sigma(2 n)},
$$

MSC (2010): Primary: 17B37; Secondary: 58A17, 15A75, 15B33, 15A15.

Keywords: $q$-determinant, $q$-permanent, $q$-Pfaffian, $q$-Hafnian, Hopf algebras

Supported by NSFC (grant nos. 11271138 and 11531004), Simons Foundation (grant 198129) and a CSC fellowship. 
where $l(\sigma)$ is the number of inversions of $\sigma \in S_{2 n}$. One can formally define the $q$-Hafnian of $B$ by replacing $q$ by $-q$ in (1.2). In general, quantum Pfaffians and Hafnians are polynomials with coefficients of rational functions in $\mathbb{Q}(q)$.

Classically the relationship between Pfaffians and Hafnians goes much deeper than formal similarity. On the enveloping algebras of the orthogonal and symplectic Lie algebras, Molev and Nazarov [MN] found the interesting correspondence between Pfaffians and Hafnians in the study of Capelli's identities. The goal of this note is to show that in the coordinate ring of two-parameter general linear quantum group $\mathrm{GL}_{r, s}(2 n)=\left\langle a_{i j},\left(\operatorname{rdet}_{r}\right)^{ \pm 1}\right\rangle$, the quantum Pfaffians satisfy the fundamental identities (Theorems 2.1 and 3.2)

$$
\begin{aligned}
\operatorname{Pf}_{r}\left(A^{T} \mathbb{J}_{s^{-1}} A\right) & =\operatorname{rdet}_{r}(A) \\
=\operatorname{cdet}_{s^{-1}}(A) & =\operatorname{Pf}_{s^{-1}}\left(A \mathbb{J}_{r} A^{T}\right),
\end{aligned}
$$

where $\operatorname{rdet}(A)(\operatorname{resp} . \operatorname{cdet}(A))$ is the quantum row (resp. column) determinant of $A=\left(a_{i j}\right)_{2 n \times 2 n}$ and

$$
\mathbb{J}_{v}=\operatorname{diag}(\underbrace{J_{v}, \cdots, J_{v}}_{n}), \quad J_{v}=\left(\begin{array}{cc}
0 & 1 \\
-v & 0
\end{array}\right) .
$$

The identities in (1.3) can be viewed as a common lift of the classical identity between Pfaffian and determinant or Hafnian and permanent. In deriving these identities, we have generalized the method of [ $\mathbf{J Z}]$ to use various $q$-forms to give a uniformed and general treatment of quantum determinants and Pfaffians as well as quantum permanents and Hafnians.

We further show that there are two cases $r= \pm s^{-1}=q$ of $\mathrm{GL}_{r, s}$ which have a simplified quantum Pfaffian over $\mathbb{Z}\left[q, q^{-1}\right]$ thanks to the $q$-Plücker relation. One special case is the usual quantum general linear group $\mathrm{GL}_{q}=$ $\mathrm{GL}_{q, q^{-1}}$, and the other is the quantum group $\mathrm{GL}_{q,-q^{-1}}$. On the second quantum group both the quantum Pfaffian and Haftnian appear and

$$
\operatorname{Pf}_{q}\left(A^{T} \mathbb{J}_{-q} A\right)=\operatorname{Hf}_{q}\left(A \mathbb{J}_{q} A^{T}\right)=\operatorname{rdet}_{q}(A) .
$$

This interesting phenomenon suggests that the quantum group $\mathrm{GL}_{q,-q^{-1}}$ provides a context where more bosonic and fermionic identities in quantum multilinear algebra may be found.

\section{Quantum determinants}

2.1. Quantum semigroup $\mathcal{A}_{r, s}$. Let $r, s$ be two fixed generic numbers in the complex field $\mathbb{C}$. The unital algebra $\mathcal{A}_{r, s}$ is an associative complex 
algebra generated by $a_{i j}, 1 \leqslant i, j \leqslant n$ subject to the relations:

$$
\begin{aligned}
& a_{i k} a_{i l}=r a_{i l} a_{i k}, \\
& a_{i k} a_{j k}=s^{-1} a_{j k} a_{i k}, \\
& r a_{i l} a_{j k}=s^{-1} a_{j k} a_{i l}, \\
& a_{i k} a_{j l}-a_{j l} a_{i k}=(r-s) a_{i l} a_{j k},
\end{aligned}
$$

where $i<j$ and $k<l$. The algebra $\mathcal{A}_{r, s}$ is a bialgebra under the comultiplication $\mathcal{A}_{r, s} \longrightarrow \mathcal{A}_{r, s} \otimes \mathcal{A}_{r, s}$ given by

$$
\Delta\left(a_{i j}\right)=\sum_{k} a_{i k} \otimes a_{k j}
$$

and the counit given by $\varepsilon\left(a_{i j}\right)=\delta_{i j}$, the Kronecker delta symbol. This bialgebra is a two-parameter quantum semigroup (cf. JL) that generalizes the quantum coordinate ring of the general linear group [FRT, [LS], which is the special case of $r=s^{-1}=q$.

The quantum row-determinant and column-determinant of $A$ are defined as follows.

$$
\begin{aligned}
\operatorname{rdet}_{r}(A) & =\sum_{\sigma \in S_{n}}(-r)^{l(\sigma)} a_{1, \sigma(1)} \cdots a_{n, \sigma(n)}, \\
\operatorname{cdet}_{s^{-1}}(A) & =\sum_{\sigma \in S_{n}}(-s)^{-l(\sigma)} a_{\sigma(1), 1} \cdots a_{\sigma(n), n} .
\end{aligned}
$$

We will show that both are group-like elements (see (2.16)-(2.17)):

$$
\begin{aligned}
\Delta\left(\operatorname{rdet}_{r}(A)\right) & =\operatorname{rdet}_{r}(A) \otimes \operatorname{rdet}_{r}(A), \\
\Delta\left(\operatorname{det}_{s^{-1}}(A)\right) & =\operatorname{cdet}_{s^{-1}}(A) \otimes \operatorname{cdet}_{s^{-1}}(A) .
\end{aligned}
$$

In fact, we have the following result $\mathbf{J L}$ (announced in $[\mathbf{T}]$ ). For our purpose, we will give a different proof using the technique of $q$-forms.

TheOREM 2.1. In the bialgebra $\mathcal{A}_{r, s}$ one has that

$$
\operatorname{rdet}_{r}(A)=\operatorname{cdet}_{s^{-1}}(A) \text {, }
$$

and moreover,

$$
\operatorname{rdet}_{r}(A) a_{i j}=(r s)^{j-i} a_{i j} \operatorname{rdet}_{r}(A) .
$$

To prove this we introduce two copies of commuting quantum exterior algebras. The first one is

$$
\Lambda(n)=\mathbb{C}\left\langle x_{1}, \ldots, x_{n}\right\rangle / I
$$

where $I$ is the ideal $\left(x_{i}^{2}, r x_{i} x_{j}+x_{j} x_{i} \mid 1 \leqslant i<j \leqslant n\right)$ and one writes $x \wedge y=$ $x \otimes y \bmod I$. Then we have that

$$
\begin{aligned}
& x_{j} \wedge x_{i}=-r x_{i} \wedge x_{j}, \\
& x_{i} \wedge x_{i}=0,
\end{aligned}
$$

where $i<j$. The algebra $\Lambda=\Lambda(n)$ is naturally $\mathbb{Z}$-graded by $\operatorname{deg}\left(x_{i}\right)=1$ and the top degree subspace is one-dimensional and spanned by $x_{1} \wedge \cdots \wedge x_{n}$. 
Assume that $a_{i j}$ 's commute with $x_{i}$ 's. We will simply write the general monomial element $a \otimes x$ as $a x$, where $a \in \mathcal{A}_{r, s}, x \in \Lambda$. Let

$$
\delta_{i}=\sum_{j=1}^{n} a_{i j} x_{j},
$$

then $\delta_{i}$ also satisfy (2.8)-(2.9). Therefore

$$
\delta_{1} \wedge \cdots \wedge \delta_{n}=\operatorname{rdet}_{r}(A) x_{1} \wedge \cdots \wedge x_{n} .
$$

The second quantum exterior algebra $\Lambda^{\prime}(n)$ is the unital associative algebra $\mathbb{C}\left\langle y_{1}, \ldots, y_{n}\right\rangle / J$, where $J$ is the ideal $\left(y_{i}^{2}, s^{-1} y_{i} y_{j}+y_{j} y_{i} \mid 1 \leqslant i<j \leqslant n\right)$. Using similar convention for $x_{i}$ 's, the relations are

$$
\begin{aligned}
& y_{j} \wedge y_{i}=-s^{-1} y_{i} \wedge y_{j}, \\
& y_{i} \wedge y_{i}=0,
\end{aligned}
$$

where $1 \leqslant i<j \leqslant n$. The algebra $\Lambda^{\prime}$ is also $\mathbb{Z}$-graded by $\operatorname{deg}\left(y_{i}\right)=1$ with the top degree 1-dimensional subspace spanned by the single vector $y_{1} \wedge \cdots \wedge y_{n}$. Assume that $y_{i}$ 's commute with $a_{i j}$ and set

$$
\partial_{i}=\sum_{j=1}^{n} a_{j i} y_{j},
$$

then $\partial_{i}$ satisfy the quantum exterior relations (2.12)-(2.13). Subsequently

$$
\partial_{1} \wedge \cdots \wedge \partial_{n}=\operatorname{cdet}_{s^{-1}}(A) y_{1} \wedge \cdots \wedge y_{n} .
$$

The algebra $\Lambda^{\prime}$ is a right $\mathcal{A}_{r, s}$-comodule with the coaction $\rho: \Lambda^{\prime} \longrightarrow$ $\Lambda^{\prime} \otimes \mathcal{A}_{r, s}$ given by $\rho\left(y_{i}\right)=\partial_{i}$. The comomule identity $(\rho \otimes \mathrm{id}) \rho=(\mathrm{id} \otimes \Delta) \rho$ leads to the following.

$$
\begin{aligned}
& (\rho \otimes \mathrm{id}) \rho\left(y_{1} \wedge \cdots \wedge y_{n}\right) \\
& =(\rho \otimes \mathrm{id}) \partial_{1} \wedge \cdots \wedge \partial_{n} \\
& =(\rho \otimes \mathrm{id}) y_{1} \wedge \cdots \wedge y_{n} \otimes \operatorname{cdet}_{s^{-1}}(A) \\
& =y_{1} \wedge \cdots \wedge y_{n} \otimes \operatorname{cdet}_{s^{-1}}(A) \otimes \operatorname{cdet}_{s^{-1}}(A)
\end{aligned}
$$

and

$$
\begin{aligned}
& (\mathrm{id} \otimes \Delta) \rho\left(y_{1} \wedge \cdots \wedge y_{n}\right) \\
& =(\operatorname{id} \otimes \Delta) y_{1} \wedge \cdots \wedge y_{n} \otimes \operatorname{cdet}_{s^{-1}}(A) \\
& =y_{1} \wedge \cdots \wedge y_{n} \otimes \Delta\left(\operatorname{cdet}_{s^{-1}}(A)\right) .
\end{aligned}
$$

Therefore

$$
\Delta\left(\operatorname{cdet}_{s^{-1}}(A)\right)=\operatorname{cdet}_{s^{-1}}(A) \otimes \operatorname{cdet}_{s^{-1}}(A) .
$$

i.e. $\operatorname{cdet}_{s^{-1}}(A)$ is a group-like element.

If we put $x=\left(x_{1}, \ldots, x_{n}\right)^{T}, y=\left(y_{1}, \ldots, y_{n}\right)^{T}, \delta=\left(\delta_{1}, \ldots, \delta_{n}\right)^{T}$ and $\partial=\left(\partial_{1}, \ldots, \partial_{n}\right)^{T}$ then

$$
\begin{gathered}
\delta=A x, \\
\partial=A^{T} y,
\end{gathered}
$$


where $A=\left(a_{i j}\right)$. Therefore $\mathcal{A}_{r, s}$ can be viewed as a set of $r$-linear and $s$ linear transformations preserving the quantum exterior algebras (2.8)-(2.9) respectively. The following is a generalization of Manin's result $\mathbf{M a}$ for the one-parameter quantum group.

Proposition 2.2. The entries of the matrix A satisfy the relations (2.1)2.4) of the quantum algebra $\mathcal{A}_{r, s}$ if and only if $\delta_{i}=\sum_{j} a_{i j} x_{j}$ and $\partial_{i}=$ $\sum_{j} a_{j i} y_{j}$ satisfy the relations (2.8)-(2.9) and (2.12)-(2.13) respectively.

We can now prove Theorem 2.1. Suppose that $\Lambda, \Lambda^{\prime}$ and $A$ commute with each other and generate a $\mathbb{Z} \times \mathbb{Z}$-bigraded algebra $\mathcal{A}=\left\langle\mathcal{A}_{r, s}, \Lambda, \Lambda^{\prime}\right\rangle=$ $\mathcal{A}_{r, s} \otimes \Lambda \otimes \Lambda^{\prime}$ with $\mathcal{A}_{(i, j)}=\mathcal{A}_{r, s} \otimes \Lambda_{i} \otimes \Lambda_{j}^{\prime}$, where $\Lambda_{i}$ (resp. $\Lambda_{i}^{\prime}$ ) is the degree $i$ subspace of $\Lambda$ (resp. $\Lambda^{\prime}$ ). Consider the following special linear element $\Phi$ in $\mathcal{A}_{r, s} \otimes \Lambda \otimes \Lambda^{\prime}$ :

$$
\Phi=\sum_{i, j=1}^{n} a_{j i} x_{i} y_{j}=x^{T} A^{T} y \in \mathcal{A}_{(1,1)} .
$$

Let $\omega_{i}=x_{i} \partial_{i}=\sum_{j=1}^{n} a_{j i} x_{i} y_{j} \in \mathcal{A}_{(1,1)}$. If follows from (2.8)-(2.9) and the commutation relations of $\partial_{i}$ that

$$
\begin{aligned}
& \omega_{i} \wedge \omega_{i}=0, \quad i=1, \ldots, n, \\
& \omega_{j} \wedge \omega_{i}=\frac{r}{s} \omega_{i} \wedge w_{j}, \quad 1 \leqslant i<j \leqslant n .
\end{aligned}
$$

Note that $\Phi=\sum_{i=1}^{n} \omega_{i}$. Using (2.21) $-(2.22)$, we have that

$$
\begin{aligned}
\wedge^{n} \Phi & =\left(\sum_{\sigma \in S_{n}}\left(\frac{r}{s}\right)^{l(\sigma)}\right) \omega_{1} \wedge \cdots \wedge \omega_{n} \\
& =[n]_{\frac{r}{s}} !\left(x_{1} \wedge \cdots \wedge x_{n}\right)\left(\partial_{1} \wedge \cdots \wedge \partial_{n}\right) \\
& =[n]_{\frac{r}{s}} ! \operatorname{cdet}_{s^{-1}}(A)\left(x_{1} \wedge \cdots \wedge x_{n}\right)\left(y_{1} \wedge \cdots \wedge y_{n}\right),
\end{aligned}
$$

where $[n]_{v} !=[n]_{v} \cdots[1]_{v},[n]_{v}=1+v+\cdots+v^{n-1}$ for any variable $v$. Similarly we can write $\Phi=\sum_{i=1}^{n} \omega_{i}^{\prime}$ with $\omega_{i}^{\prime}=\delta_{i} y_{i}=\sum_{j=1}^{n} a_{i j} x_{j} y_{i}$. It is easy to see that the elements $\omega_{i}^{\prime}$ satisfy the same quantum exterior algebra (2.21)-(2.22). Thus

$$
\begin{aligned}
\wedge^{n} \Phi & =[n]_{\frac{r}{s}} ! \omega_{1}^{\prime} \wedge \cdots \wedge \omega_{n}^{\prime} \\
& =[n]_{\frac{r}{s}} !\left(\delta_{1} \wedge \cdots \wedge \delta_{n}\right)\left(y_{1} \wedge \cdots \wedge y_{n}\right) \\
& =[n]_{\frac{r}{s}} \operatorname{lrdet}_{r}(A)\left(x_{1} \wedge \cdots \wedge x_{n}\right)\left(y_{1} \wedge \cdots \wedge y_{n}\right) .
\end{aligned}
$$

Subsequently we get the first identity of Theorem 2.1:

$$
\operatorname{rdet}_{r}(A)=\operatorname{cdet}_{s^{-1}}(A) \text {. }
$$

For a pair of $t$ indices $i_{1}, \ldots, i_{t}$ and $j_{1}, \ldots, j_{t}$, we define the quantum row-minor determinant $\operatorname{det}_{r}\left(A_{j_{1} \ldots j_{t}}^{i_{1} \ldots i_{t}}\right)$ as in (2.6). Then

$$
\delta_{i_{1}} \wedge \cdots \wedge \delta_{i_{t}}=\sum_{j_{1}<\cdots<j_{t}} \operatorname{rdet}_{r}\left(A_{j_{1} \ldots j_{t}}^{i_{1} \ldots i_{t}}\right) x_{j_{1}} \wedge \cdots \wedge x_{i_{t}},
$$


which implies that $\operatorname{det}_{r}\left(A_{j_{1} \ldots j_{t}}^{i_{1} \ldots i_{t}}\right)=0$ whenever there are two identical rows.

For indices $1 \leqslant i_{1}<\cdots<i_{t}, i_{t+1}<\cdots<i_{n} \leqslant n$, it is readily seen that

$$
\delta_{i_{1}} \wedge \cdots \wedge \delta_{i_{t}} \wedge \delta_{i_{t+1}} \wedge \cdots \wedge \delta_{i_{n}}=(-r)^{i_{1}+\cdots+i_{t}-\frac{t(t+1)}{2}} \delta_{1} \wedge \cdots \wedge \delta_{n} .
$$

Note that $x_{j}$ 's also satisfy the same wedge relations. These then imply the following Laplace expansion by invoking (2.23):

$$
\operatorname{rdet}_{r}(A)=\sum_{\substack{j_{1}<\cdots<j_{t} \\ j_{t+1}<\cdots<j_{n}}}(-r)^{\left(j_{1}+\cdots+j_{t}\right)-\left(i_{1}+\cdots+i_{t}\right)} \operatorname{rdet}_{r}\left(A_{j_{1} \ldots j_{t}}^{i_{1} \ldots i_{t}}\right) \operatorname{rdet}_{r}\left(A_{j_{t+1} \ldots j_{n}}^{i_{t+1} \ldots i_{n}}\right) .
$$

In particular, for fixed $i, k$

$$
\operatorname{rdet}_{r}(A) \delta_{i k}=\sum_{j=1}^{n}(-r)^{j-i} a_{i j} \operatorname{rdet}_{r}\left(A_{\hat{j}}^{\hat{k}}\right)=\sum_{j=1}^{n}(-r)^{i-j} \operatorname{rdet}_{r}\left(A_{\hat{j}}^{\hat{k}}\right) a_{i j},
$$

where $\hat{i}$ means the omission of $i$ and here we use it to denote the indices $1, \ldots, i-1, i+1, \ldots, n$ for brevity.

As for the quantum (column) determinant or column-minor, we also have that $\operatorname{cdet}_{s^{-1}}\left(A_{j_{1} \ldots j_{t}}^{i_{1} \ldots i_{t}}\right)=0$ whenever there are two identical columns. The corresponding Laplace expansion for a permutation $\left(i_{1} \ldots i_{n}\right)$ of $n$ such that $i_{1}<\cdots<i_{r}, i_{r+1}<\cdots<i_{n}$

$$
=\sum_{\substack{j_{1}<\cdots<j_{r} \\ j_{r+1}<\cdots<j_{n}}}^{\operatorname{cdet}_{s^{-1}}(A)}(-s)^{\left(i_{1}+\cdots+i_{r}\right)-\left(j_{1}+\cdots+j_{r}\right)} \operatorname{cdet}_{s^{-1}}\left(A_{i_{1} \ldots i_{r}}^{j_{1} \ldots j_{r}}\right) \operatorname{cdet}_{s^{-1}}\left(A_{i_{r+1} \ldots i_{n}}^{j_{r+1} \ldots j_{n}}\right) .
$$

In particular, we have that for fixed $i, k$

$$
\begin{aligned}
\operatorname{cdet}_{s^{-1}}(A) \delta_{i k} & =\sum_{j=1}^{n}(-s)^{i-j} a_{j i} \operatorname{cdet}_{s^{-1}}\left(A_{\hat{k}}^{\hat{j}}\right) \\
& =\sum_{j=1}^{n}(-s)^{j-i} \operatorname{cdet}_{s^{-1}}\left(A_{\hat{k}}^{\hat{j}}\right) a_{j i} .
\end{aligned}
$$

Like the determinant, the minor row determinant is also equal to the column minor determinant for any pairs of ordered indices $1 \leqslant i_{1}<\cdots<$ $i_{t} \leqslant n$ and $1 \leqslant j_{1}<\cdots<j_{t} \leqslant n$

$$
\operatorname{rdet}_{r}\left(A_{j_{1} \ldots j_{t}}^{i_{1} \ldots i_{t}}\right)=\operatorname{cdet}_{s^{-1}}\left(A_{j_{1} \ldots j_{t}}^{i_{1} \ldots i_{t}}\right) .
$$

Let $D_{v}=\operatorname{diag}\left(v, v^{2}, \ldots, v^{n}\right)$, and define the inner automorphism $\tau$ of $\operatorname{Mat}_{n}\left(\mathcal{A}_{r, s}\right)$ via conjugation by $D_{v}$ as follows: for any $C=\left(c_{i j}\right) \in \operatorname{Mat}_{n}\left(\mathcal{A}_{r, s}\right)$

$$
C_{v}^{\tau}=D_{v}^{-1} C D_{v}=\left(v^{j-i} c_{i j}\right) .
$$

Let $\operatorname{adj}(A)=\left((-1)^{i-j} \operatorname{rdet}_{r}\left(A_{\hat{i}}^{\hat{j}}\right)\right)$ be the quantum adjoint matrix associated to $A$. Then the Laplace expansions (2.24)-(2.25) give the first version of the quantum Cramer's rule in $\mathcal{A}_{r, s}$. 
Proposition 2.3. Over the quantum semigroup $\mathcal{A}_{r, s}(n)$, one has that

$$
\begin{aligned}
A_{r}^{\tau} \operatorname{adj}(A) & =\operatorname{adj}(A) A_{s^{-1}}^{\tau}=\operatorname{rdet}_{r}(A) I, \\
A(\operatorname{adj}(A))_{r^{-1}}^{\tau} & =(\operatorname{adj}(A))_{s}^{\tau} A=\operatorname{rdet}_{r}(A) I,
\end{aligned}
$$

where $I$ is the identity matrix of size $n$.

Using the quantum Cramer's rule, we have that

$$
A_{r}^{\tau} \operatorname{rdet}_{r}(A)=A_{r}^{\tau} \operatorname{adj}(A) A_{s^{-1}}^{\tau}=\operatorname{rdet}_{r}(A) A_{s^{-1}}^{\tau},
$$

therefore $a_{i j} \operatorname{det}_{r}(A)=(r s)^{i-j} \operatorname{det}_{r}(A) a_{i j}$, which is the second identity in Theorem 2.1.

2.2. Quantum group $\mathbf{G L}_{r, s}(n)$. The second identity in Theorem 1.3 implies that $\operatorname{rdet}_{r}(A)$ is a regular element in the $\operatorname{ring} \mathcal{A}_{r, s}$, therefore we can define the localization $\mathcal{A}_{r, s}\left[\operatorname{det}_{r}^{-1}\right]$, which will be denoted as $\mathrm{GL}_{r, s}(n)$.

Although in general $A_{r}^{\tau} \neq A_{s^{-1}}^{\tau}$, this does not prevent the quantum Cramer's rule (2.27) from giving an inverse element for $A$ in $\operatorname{Mat}_{n}\left(\mathrm{GL}_{r, s}\right)$. In fact, (2.29) gives the following identity:

$$
\left(\operatorname{rdet}_{r}(A)\right)^{-1} A_{r}^{\tau}=A_{s^{-1}}^{\tau}\left(\operatorname{rdet}_{r}(A)\right)^{-1} .
$$

So the quantum Cramer's rule can be rewritten as

Proposition 2.4. On the quantum group $G L_{r, s}$ one has that

$$
\begin{aligned}
\left(\operatorname{rdet}_{r}(A)\right)^{-1} A_{r}^{\tau} \cdot \operatorname{adj}(A) & =\operatorname{adj}(A) \cdot A_{s^{-1}}^{\tau}\left(\operatorname{rdet}_{r}(A)\right)^{-1}=I, \\
A \cdot \operatorname{adj}(A)_{r^{-1}}^{\tau}\left(\operatorname{rdet}_{r}(A)\right)^{-1} & =\left(\operatorname{rdet}_{r}(A)\right)^{-1} \operatorname{adj}(A)_{s}^{\tau} \cdot A=I,
\end{aligned}
$$

or equivalently in $\operatorname{Mat}_{n}\left(\mathrm{GL}_{r, s}\right)$

$$
A^{-1}=\operatorname{rdet}_{r}(A)^{-1} \operatorname{adj}(A)_{s}^{\tau}=\operatorname{adj}(A)_{r^{-1}}^{\tau} \operatorname{rdet}_{r}(A)^{-1} .
$$

The second identity (2.32) is obtained from (2.31) by applying the automorphism $\tau^{-1}$ associated to $D_{r}^{-1}$ or $D_{s}$ to both sides respectively.

By defining the antipode

$$
\begin{aligned}
S\left(a_{i j}\right) & =(-s)^{j-i} \operatorname{cdet}_{s^{-1}}(A)^{-1} \operatorname{cdet}_{s^{-1}}\left(A_{\hat{i}}^{\hat{j}}\right) \\
& =(-r)^{i-j} \operatorname{rdet}_{r}\left(A_{\hat{i}}^{\hat{j}}\right) \operatorname{rdet}_{r}(A)^{-1}
\end{aligned}
$$

the bialgebra $\mathrm{GL}_{r, s}=\mathcal{A}_{r, s}\left[\operatorname{rdet}_{r}^{-1}\right]$ becomes a Hopf algebra, thus a quantum group in the sense of Drinfeld. In fact, $A S(A)=S(A) A=I$ follows from Proposition 2.4, therefore (id $\otimes S) \Delta=(S \otimes \mathrm{id}) \Delta=\varepsilon$.

We remark that the quantum semigroup $\mathcal{A}_{r, s}$ offers new identities to quantum multilinear algebra studied in [JZ (see [LT] for several interesting identities in the classical situation; also see [TT, $\mathbf{H H}, \mathbf{I W}, \mathbf{Z}, \mathbf{G}]$ for the usual quantum group $\mathrm{GL}_{q}$ ). Moreover, if $r, s$ are indeterminates over the field $F$ of characteristic 0 , then the quantum group $\mathcal{A}_{r, s}$ can be defined over the rational field $F(r, s)$, and all results in the paper hold in that situation. 


\section{Quantum Pfaffians and Hafnians}

3.1. $r$-Pfaffians. Let $B=\left(b_{i j}\right)$ be a $2 n \times 2 n$ square matrix with noncommutative entries, and let $\mathcal{B}$ be the associative algebra generated by $b_{i j}, i<j$. Assume that $b_{i j}$ commute with the algebra $\Lambda=\Lambda(2 n)$ generated by $x_{1}, \ldots, x_{2 n}$, and consider the algebra $\mathcal{B} \otimes \Lambda$.

Let $\Omega=\sum_{1 \leqslant i<j \leqslant 2 n} b_{i j}\left(x_{i} \wedge x_{j}\right) \in \mathcal{B}_{q} \otimes \Lambda$. The quantum Pfaffian $\operatorname{Pf}_{r}(B)$ is defined by

$$
\wedge^{n} \Omega=[n]_{r^{4}} ! \operatorname{Pf}_{r}(B) x_{1} \wedge x_{2} \wedge \cdots \wedge x_{2 n} .
$$

Explicitly the quantum Pfaffian is given by $[\mathbf{J Z}]$ :

$$
\operatorname{Pf}_{r}(B)=\frac{1}{[n]_{r^{4}} !} \sum_{\sigma \in \Pi^{\prime}}(-r)^{l(\sigma)} b_{\sigma(1) \sigma(2)} b_{\sigma(3) \sigma(4)} \cdots b_{\sigma(2 n-1) \sigma(2 n)},
$$

where $\Pi^{\prime}$ is the set of permutations $\sigma$ of $2 n$ such that $\sigma(1)<\sigma(2), \ldots, \sigma(2 n-$ 1) $<\sigma(2 n)$.

In the quantum coordinate ring $\mathcal{A}_{r, s}(2 n)$, we set for $1 \leqslant i, j \leqslant 2 n$

$$
b_{i j}=\sum_{m=1}^{n} \operatorname{cdet}_{s^{-1}}\left(A_{i, j}^{2 m-1,2 m}\right)=\sum_{m=1}^{n}\left(a_{2 m-1, i} a_{2 m, j}-s^{-1} a_{2 m, i} a_{2 m-1, j}\right) .
$$

The matrix $B=\left(b_{i j}\right)$ can be compactly written as

$$
B=A^{T} \mathbb{J}_{s^{-1}} A
$$

where for any variable $s$

$$
\mathbb{J}_{s^{-1}}=\mathbb{J}_{s^{-1}}(n)=\operatorname{diag}(\underbrace{J_{s^{-1}}, \cdots, J_{s^{-1}}}_{n}), \quad J_{s^{-1}}=\left(\begin{array}{cc}
0 & 1 \\
-s^{-1} & 0
\end{array}\right) .
$$

The special case of the following result at $r=s^{-1}=q$ was proved [JR under a stronger assumption using representation theory, and later obtained via the quantum exterior algebra [JZ. We will derive the generalization under a weaker assumption.

Theorem 3.1. On the quantum coordinate ring $\mathcal{A}_{r, s}(2 n)$ one has that

$$
\operatorname{Pf}_{r}\left(A^{T} \mathbb{J}_{s^{-1}} A\right)=\operatorname{rdet}_{r}(A),
$$

where $A^{T} \mathbb{J}_{s^{-1}} A=\left(b_{i j}\right)$ and $b_{i j}$ are defined by (3.3).

Proof. Note that for $1 \leqslant i<j \leqslant 2 n$

$$
b_{i j}=\sum_{m=1}^{n} \operatorname{rdet}_{r}\left(A_{i, j}^{2 m-1,2 m}\right)=\sum_{m=1}^{n}\left(a_{2 m-1, i} a_{2 m, j}-r a_{2 m-1, j} a_{2 m, i}\right) .
$$

With the $b_{i j}$, by the quantum exterior relations of $x_{i}$ 's it follows that the 2 -form $\Omega$ can also be written as

$$
\Omega=\delta_{1} \wedge \delta_{2}+\delta_{3} \wedge \delta_{4}+\cdots+\delta_{2 n-1} \wedge \delta_{2 n},
$$


where $\delta_{i}=\sum_{j=1}^{2 n} a_{i j} x_{j}(i=1,2, \ldots, 2 n)$ and they obey (2.8) and (2.9). Subsequently

$$
\begin{aligned}
& \left(\delta_{2 j-1} \wedge \delta_{2 j}\right)\left(\delta_{2 i-1} \wedge \delta_{2 i}\right)=r^{4}\left(\delta_{2 i-1} \wedge \delta_{2 i}\right)\left(\delta_{2 j-1} \wedge \delta_{2 j}\right), i<j \\
& \left(\delta_{2 i-1} \wedge \delta_{2 i}\right)^{2}=0
\end{aligned}
$$

Therefore

$$
\wedge^{n} \Omega=[n]_{r^{4}} ! \delta_{1} \wedge \cdots \wedge \delta_{2 n}=[n]_{r^{4}} \operatorname{lrdet}_{r}(A) x_{1} \wedge \cdots \wedge x_{2 n},
$$

where the last identity uses the wedge formulation of $\operatorname{det}_{r}(A)$ in $\mathcal{A}_{r, s}(2 n)$. Hence $\operatorname{det}_{r}(A)=\operatorname{Pf}_{r}(B)$.

3.2. $s^{-1}$-Pfaffians. Similar to the $r$-Pfaffian, we utilize another 2 -form to define the $s^{-1}$-Hafnian in $\mathcal{B}$. Let

$$
\Omega^{\prime}=\sum_{1 \leqslant i<j \leqslant 2 n} b_{i j} y_{i} \wedge y_{j},
$$

where $y_{i}$ satisfy the quantum exterior relations (2.12)-(2.13) and commute with $b_{i j}$ 's. The $s^{-1}$-Hafnian of $B$ is then defined by

$$
\wedge^{n} \Omega^{\prime}=[n]_{s^{-4}} ! \mathrm{Pf}_{s^{-1}}(B) y_{1} \wedge \cdots \wedge y_{2 n} .
$$

Using the quantum exterior relations, we have the explicit formula:

$$
\mathrm{Pf}_{s^{-1}}(B)=\frac{1}{[n]_{s^{-4}} !} \sum_{\sigma \in \Pi^{\prime}}(-s)^{-l(\sigma)} b_{\sigma(1) \sigma(2)} b_{\sigma(3) \sigma(4)} \cdots b_{\sigma(2 n-1) \sigma(2 n)} .
$$

We also have the second identity between the quantum Pfaffian and quantum determinant.

THEOREM 3.2. In the quantum coordinate ring $\mathcal{A}_{r, s}(2 n)$ we have

$$
\mathrm{Pf}_{s^{-1}}\left(A \mathbb{J}_{r} A^{T}\right)=\operatorname{cdet}_{s^{-1}}(A) .
$$

where $A \rrbracket_{r} A^{T}=\left(b_{i j}^{\prime}\right)_{2 n \times 2 n}$ and for $i<j$

$$
b_{i j}^{\prime}=\sum_{m=1}^{n} \operatorname{rdet}_{r}\left(A_{2 m-1,2 m}^{i, j}\right)=\sum_{m=1}^{n}\left(a_{i, 2 m-1} a_{j, 2 m}-r a_{i, 2 m} a_{j, 2 m-1}\right) .
$$

Proof. This is proved by a similar method to that of Theorem 3.1. In fact, note that the 2 -form $\Omega^{\prime}$ can be rewritten as

$$
\Omega^{\prime}=\partial_{1} \wedge \partial_{2}+\partial_{3} \wedge \partial_{4}+\cdots+\partial_{2 n-1} \wedge \partial_{2 n},
$$

where $\partial_{i}=\sum_{j=1}^{2 n} a_{j i} x_{j}(i=1,2, \ldots, 2 n)$, and $\partial_{i}$ 's obey the quantum exterior algebra (2.31)-(2.32). Therefore

$$
\begin{aligned}
\wedge^{n} \Omega^{\prime} & =[n]_{s^{-4}} ! \partial_{1} \wedge \cdots \wedge \partial_{2 n} \\
& =[n]_{s^{-4}} ! \operatorname{cdet}_{s^{-1}}(A) y_{1} \wedge y_{2} \wedge \cdots \wedge y_{2 n},
\end{aligned}
$$

which implies that $\operatorname{cdet}_{s^{-1}}(A)=\operatorname{Pf}_{s^{-1}}\left(B^{\prime}\right)$.

Since $\operatorname{rdet}_{r}(A)=\operatorname{cdet}_{s^{-1}}(A)$ on the quantum semigroup $\mathcal{A}_{r, s}(2 n)$, we have the following result. 
Theorem 3.3. For $i<j$, let $b_{i j}=\sum_{m=1}^{n} \operatorname{cdet}_{s^{-1}}\left(A_{i, j}^{2 m-1,2 m}\right)$ and $b_{i j}^{\prime}=$ $\sum_{\text {that }}^{n} \operatorname{rdet}_{r}\left(A_{2 m-1,2 m}^{i, j}\right)$ in the quantum semigroup $\mathcal{A}_{r, s}(2 n)$, then one has

$$
\mathrm{Pf}_{r}(B)=\mathrm{Pf}_{s^{-1}}\left(B^{\prime}\right) .
$$

3.3. Quantum Pfaffians and Hafnians. When $r= \pm s^{-1}=q$, the quantum Pfaffian has a simplified expression and is an element of $\mathrm{GL}_{q, \pm q^{-1}}$ with coefficients in $\mathbb{Z}\left[q, q^{-1}\right]$. The case of $r=s^{-1}=q$ reduces to the usual quantum group $\mathrm{GL}_{q}$ and was discussed in details in [JZ], while $r=-s^{-1}$ gives rise to another quantum group on which both the quantum Pfaffian and Hafnian appear. We focus on this special case of $r=-s^{-1}=q$.

Let $\overline{\mathcal{B}}_{q}$ be the unital associative algebra generated by $b_{i j}, 1 \leqslant i<j \leqslant 2 n$ subject to the quadratic relations

$$
b_{i j} b_{k l}-q b_{i k} b_{j l}+q^{2} b_{i l} b_{j k}=b_{k l} b_{i j}-q^{-1} b_{j l} b_{i k}+q^{-2} b_{j k} b_{i l},
$$

for $1 \leqslant i<j<k<l \leqslant 2 n$. We refer them as the $q$-Maya relations or $q$-Plücker relations, since the special case of $q=1$ can be recast as a Young diagram identity connected to Maya diagrams $[\mathbf{H}]$.

First of all, using a similar method of [JZ] we have the following simplification of the quantum Pfaffian.

Proposition 3.4. On the algebra $\overline{\mathcal{B}}_{q}$, the quantum Pfaffian $\operatorname{Pf}_{q}$ is simply given by

$$
\operatorname{Pf}_{q}(B)=\sum_{\sigma \in \Pi}(-q)^{l(\sigma)} b_{\sigma(1) \sigma(2)} b_{\sigma(3) \sigma(4)} \cdots b_{\sigma(2 n-1) \sigma(2 n)},
$$

where $\Pi$ is the set of permutations $\sigma \in S_{2 n}$ such that $\sigma(1)<\sigma(2), \ldots, \sigma(2 n-$ $1)<\sigma(2 n)$ and $\sigma(1)<\sigma(3)<\cdots<\sigma(2 n-1)$.

By a direct computation as in [JZ, the elements $b_{i j}$ 's given in (3.3) can be shown to satisfy the $q$-Maya relations. We remark that the $b_{i j}$ defined by (3.3) in the two-parameter quantum semigroup $\mathcal{A}_{r, s}$ does not satisfy the quantum Maya relation unless $r^{2} s^{2}=1$. Moreover, the quantum Pfaffian can also be computed iteratively as follows. A proof can be similarly given as in $[\mathbf{J Z}]$.

Proposition 3.5. On the algebra $\overline{\mathcal{B}}_{q}$ we have

$$
\operatorname{Pf}_{q}(B)=\sum_{j=2}^{2 n}(-q)^{j-2} \operatorname{Pf}_{q}\left(B_{1 j}^{1 j}\right) \operatorname{Pf}_{q}\left(B_{2, \ldots, \hat{j}, \ldots, 2 n}^{2, \ldots, \hat{j}, \ldots, 2 n}\right) .
$$

Let $\overline{\mathcal{B}}_{q}^{\prime}$ be the algebra generated by $b_{i j}^{\prime}$ for $1 \leqslant i<j \leqslant 2 n$ modulo the ideal generated by the $(-q)$-Maya relations

$$
b_{i j}^{\prime} b_{k l}^{\prime}+q b_{i k}^{\prime} b_{j l}^{\prime}+q^{2} b_{i l}^{\prime} b_{j k}^{\prime}=b_{k l}^{\prime} b_{i j}^{\prime}+q^{-1} b_{j l}^{\prime} b_{i k}^{\prime}+q^{-2} b_{j k}^{\prime} b_{i l}^{\prime},
$$

where $1 \leqslant i<j<k<l \leqslant 2 n$. 
Similar to the quantum determinant and Pfaffian, we introduce the quantum (column)-permanent and Hafnian by

$$
\begin{aligned}
\operatorname{per}_{q}(A) & =\sum_{\sigma \in S_{n}} q^{l(\sigma)} a_{\sigma(1), 1} a_{\sigma(2), 2} \cdots a_{\sigma(n), n} \\
\operatorname{Hf}_{q}(B) & =\frac{1}{[n]_{q^{4}} !} \sum_{\sigma \in \Pi^{\prime}} q^{l(\sigma)} b_{\sigma(1) \sigma(2)} b_{\sigma(3) \sigma(4)} \cdots b_{\sigma(2 n-1) \sigma(2 n)} .
\end{aligned}
$$

Similar to the quantum Pfaffian, we can simplify the definition of the quantum Hafnian.

Proposition 3.6. On the algebra $\overline{\mathcal{B}}_{q}^{\prime}$, the quantum Hafnian $\mathrm{Hf}_{q}$ is simply given by

$$
\operatorname{Hf}_{q}\left(B^{\prime}\right)=\sum_{\sigma \in \Pi} q^{l(\sigma)} b_{\sigma(1) \sigma(2)}^{\prime} b_{\sigma(3) \sigma(4)}^{\prime} \cdots b_{\sigma(2 n-1) \sigma(2 n)}^{\prime},
$$

where $\Pi=\left\{\sigma \in S_{2 n} \mid \sigma(2 i-1)<\sigma(2 i), \sigma(1)<\sigma(3)<\cdots<\sigma(2 n-1)\right\}$.

Then $\mathrm{Hf}_{q}$ can be evaluated inductively as follows.

$$
\operatorname{Hf}_{q}\left(B^{\prime}\right)=\sum_{j=2}^{2 n} q^{j-2} \operatorname{Hf}_{q}\left(B_{1 j}^{\prime 1 j}\right) \operatorname{Hf}_{q}\left(\begin{array}{c}
B_{2, \ldots, \hat{j}, \ldots, 2 n}^{2, \ldots, \hat{j}, \ldots, 2 n} \\
2 .
\end{array}\right)
$$

Moreover, when the matrix elements $b_{i j}^{\prime}$ are special quadratic elements in $\mathrm{GL}_{q,-q^{-1}}$, we can also express $\mathrm{Hf}_{q}$ in terms of per $_{q}$. The following is an immediate consequence of Theorem 3.2 .

Proposition 3.7. In the quantum coordinate ring $\mathcal{A}_{q,-q^{-1}}(2 n)$ we have

$$
\operatorname{Hf}_{q}\left(A \rrbracket_{q} A^{T}\right)=\operatorname{per}_{q}(A),
$$

where $A \rrbracket_{q} A^{T}=B^{\prime}$ is given by $(i<j)$

$$
b_{i j}^{\prime}=\sum_{m=1}^{n} \operatorname{per}_{q}\left(A_{2 m-1,2 m}^{i, j}\right)=\sum_{m=1}^{n}\left(a_{i, 2 m-1} a_{j, 2 m}+q a_{j, 2 m-1} a_{i, 2 m}\right) .
$$

\section{Acknowledgments}

The first author would like to thank Alex Molev for stimulating discussions on related topics. We also thank Ken Goodearl for his interest on the paper. The authors are grateful to the referee for encouragement to upgrade the results from the one-parameter quantum group to the two-parameter analogue.

\section{References}

[AST] M. Artin, W. Schelter, J. Tate, Quantum deformations of $\mathrm{GL}_{n}$, Comm. Pure Appl. Math. 44 (1991), 879-895.

[C] E. R. Caianiello, Combinatorics and renormalization in quantum field theory, Frontiers in Physics, W. A. Benjamin, Inc., Reading-London-Amsterdam, 1973. 
[FRT] L. D. Faddeev, N. Yu. Reshetikhin, L. A. Takhtajan, Quantization of Lie groups and Lie algebras, Algebraic analysis, Vol. I, pp. 129-139, Academic Press, Boston, MA, 1988.

[G] K. R. Goodearl, Commutation relations for arbitrary quantum minors, Pac. J. Math. 228 (2006), 63-102.

[HH] M. Hashimoto, T. Hayashi, Quantum multilinear algebra. Tohoku Math. J. (2) 44 (1992), 471-521.

[H] R. Hirota, The direct method in soliton theory, English transl. by A. Nagai, J. Nimmo, C. Gilson from 1992 Japanese ed., Cambridge University Press, Cambridge, 2004.

[IW] M. Ishikawa, M. Wakayama, Minor summation formula of Pfaffians, Lin. Multilin. Algebra 39 (1995), 285-305.

[JL] N. Jing, M. Liu, R-matrix realization of two-parameter quantum group $U_{r, s}\left(\mathfrak{g l}_{n}\right)$, Commun. Math. Stat. 2 (2014), 211-230.

[JR] N. Jing, R. Ray, Zonal polynomials and quantum antisymmetric matrices. Bull. Inst. Math. Acad. Sinica (N.S.) 7 (2012), 1-31.

[JZ] N. Jing, J. Zhang, Quantum Pfaffians and hyper-Pfaffians, Adv. Math. 265 (2014), $336-361$.

[LS] T. Levasseur, J. T. Stafford, The quantum coordinate ring of the special linear group, J. Pure Appl. Algebra 86 (1993), 181-186.

[LT] J.-G. Luque, J. Y. Thibon, Pfaffian and Hafnian identities in shuffle algebras, Adv. Appl. Math. 29 (2002), 620-646.

[Ma] Yu. I. Manin, Notes on quantum groups and quantum de Rham complexes. Teoret. Mat. Fiz. 92 (1992), 425-450; English transl. in: Theoret. Math. Phys. 92 (1992), 997-1023.

[M] H. Minc, Permanents, Encyclopedia of Mathematics and its Applications, Vol. 6, Addison-Wesley Publishing Co., Reading, Mass., 1978.

[MN] A. Molev, M. Nazarov, Capelli identities for classical Lie algebras, Math. Ann. 313 (1999), 315-357.

[T] M. Takeuchi, A two-parameter quantization of GL(n), Proc. Jpn. Acad. 66 (Ser. A) (1990), 112-114.

[TT] E. Taft, J. Towber, Quantum deformation of flag schemes and Grassmann schemes. I. A q-deformation of the shape-algebra for GL(n), J. Algebra 142 (1991), 1-36.

[V] D. Vere-Jones, A generalization of permanents and determinants, Lin. Algebra Appl. 111 (1988), 119-124.

[Z] J. J. Zhang, The quantum Cayley-Hamilton theorem, J. Pure Appl. Algebra 129 (1998), 101-109.

NJ: Department of Mathematics, North Carolina State University, Raleigh, NC 27695, USA

E-mail address: jing@math.ncsu.edu

JZ: Institute of Mathematics and Statistics, University of Sao Paulo, Sao

PAUlO, BrazIL 05315-970

E-mail address: j.zhang1792@gmail.com 